\title{
Influence of NQO1 Polymorphisms on Warfarin Maintenance Dose: A Systematic Review and Meta-Analysis (rs1800566 and rs10517)
}

\author{
Lihong Tian $\mathbb{D}^{1},{ }^{1}$ Pingping Xiao, ${ }^{2}$ Bingrong Zhou, ${ }^{1}$ Yishan Chen, ${ }^{1}$ Lijuan Kang, \\ Qingqing Wang, ${ }^{2}$ Jianfeng Lin, ${ }^{1}$ Min Son, ${ }^{1}$ and Qingxiu $\mathrm{Wu}^{1}$ \\ ${ }^{1}$ Department of General Medicine, The Second Affiliated Hospital of Xiamen Medical College, Xiamen 361021, China \\ ${ }^{2}$ Department of Hematology/Rheumatology, The Second Affiliated Hospital of Xiamen Medical College, Xiamen 361021, China
}

Correspondence should be addressed to Lihong Tian; doctortlh@163.com

Received 6 January 2021; Revised 15 July 2021; Accepted 4 August 2021; Published 12 August 2021

Academic Editor: Rosario D. C. Hirata

Copyright (C) 2021 Lihong Tian et al. This is an open access article distributed under the Creative Commons Attribution License, which permits unrestricted use, distribution, and reproduction in any medium, provided the original work is properly cited.

\begin{abstract}
This meta-analysis was conducted to analyze the effect of NQO1 polymorphism on the warfarin maintenance dosage. Using strict inclusion and exclusion criteria, we searched PubMed, EMBASE, and the Cochrane Library for eligible studies published prior to July 7, 2021. The required data were extracted, and experts were consulted when necessary. Review Manager Version 5.4 software was used to analyze the relationship between NQO1 polymorphisms and the warfarin maintenance dosage. Four articles involving 757 patients were included in the meta-analysis. Patients who were NQO1 rs10517 G carriers (AG carriers or GG carriers) required a $48 \%$ higher warfarin maintenance dose than those who were AA carriers. Patients with NQO1 rs1800566 CT carriers required a $13 \%$ higher warfarin dose than those who were CC carriers, with no associations observed with the other comparisons of the NQO1 rs1800566 genotypes. However, the results obtained by comparing the NQO1 rs1800566 genotypes require confirmation, as significant changes in the results were found in sensitivity analyses. Our meta-analysis suggests that the NQO1 rs10517and NQO1 rs1800566 variant statuses affect the required warfarin maintenance dose.
\end{abstract}

\section{Introduction}

Warfarin is an oral anticoagulant, widely used for the treatment and prevention of thromboembolic events owing to its anticoagulant efficacy and economic applicability, particularly in patients in developing countries. However, it has some disadvantages, such as a narrow therapeutic range, high interindividual variability in the maintenance dosage, and the risk of adverse effects, including the recurrence of thromboembolism and fatal bleeding risk [1].

Previous studies have shown that gene polymorphisms, including those in CYP2C9, CYP4F2, VKORC1, and GGCX, are associated with warfarin dosage adjustment [2-7]. In addition, clinical characteristics, such as height, body weight, and target International Normalized Ratio value, can influence the warfarin maintenance dosage [8-10]. However, these factors do not account for all current differences in warfarin dosage requirements among individuals and ethnicities
[11]. Genetic polymorphism in addition to clinical factors can account for approximately $50 \%$ of the variability in the warfarin dosage requirements $[12,13]$, and NQO1 genotypes may additionally explain the variance in the therapeutic warfarin dosage [14]. Moreover, a significant relationship between the NQO1 (rs1800566 and rs15017) genotype and warfarin dosage has been observed $[15,16]$; therefore, NQO1 may contribute to warfarin dosage variation.

NQO1 is located on chromosome 16 and encodes $\mathrm{NAD}(\mathrm{P}) \mathrm{H}$ dehydrogenase quinone 1 , an enzyme that catalyzes the reduction of quinones, including vitamin $K$ [14]. During vitamin $\mathrm{K}$ recycling, vitamin $\mathrm{K}$ is reduced to vitamin $\mathrm{K}$ hydroquinone by NQO1. Studies have reported that individuals carrying both mutated genomic alleles have no NQO1 activity, whereas individuals heterozygous with one mutated allele have low-to-intermediate NQO1 activity compared with wild-type individuals $[17,18]$. Warfarin exerts its action by interfering with the cyclic interconversion of 
vitamin K to vitamin K epoxide [19]. Therefore, NQO1 activity is associated with the enzyme's capacity to reduce vitamin $\mathrm{K}$ and the concentration of dicoumarol required to inhibit NQO1 activity [20]. Since the discovery of NQO1, various in vitro and in vivo studies have reported that NQO1 polymorphisms are important for maintaining vitamin $\mathrm{K}$ reduction in the absence of vitamin $\mathrm{K}$ epoxide reductase (VKOR) [21-23]. Previous studies showed that NQO1 rs1800566 variants decrease the coagulation ability and ischemic stroke risk compared with the wild-type variant [24]. Therefore, NQO1 rs1800566 polymorphisms likely influence the required dosage of warfarin. Momary et al. [25] focused on the effect of NQO1 rs1800566 on warfarin dosage requirements. Subsequently, many studies of various patient populations have focused on the relationship between NQO1 and warfarin dosage requirements and found a significant difference in the warfarin dosages among diverse NQO1 genotypes [9, 15, $16,25]$. However, the role of diverse NQO1 genotypes is controversial. Chung et al. reported that among Korean people, NQO1 rs1800566 variant homozygote carriers required lower stable warfarin dosages than those with the wild-type allele [9]; El Rouby et al. indicated that NQO1 rs1800566 variants were associated with higher dosage requirements among Latino populations [15]. No associations have been identified between NQO1 rs1800566 and warfarin in populations of African or European ancestry $[25,26]$. The role of NQO1 genotypes may differ in people of different ethnicities. Although previous meta-analyses confirmed the effects of CYP2C9, VORCK1, GGCX, and CYP4F2 genotypes on the warfarin dosage [2-6], studies of the influence of NQO1 on warfarin dosage are limited. Therefore, we investigated the effects of individual NQO1 genotypes on the mean daily warfarin dosage (MDWD) using meta-analyses.

\section{Materials and Methods}

2.1. Search Strategy. The search for eligible studies published in English prior to July 7, 2021, was conducted on PubMed, EMBASE, and the Cochrane Library. The search strategy used the following keywords: (NQO1 OR "nicotinamide adenine dinucleotide phosphate, reduced quinone oxidoreductase") AND (warfarin) AND (gene OR genotype OR genetics OR alleles OR polymorphism OR pharmacogenetics). The included literature references were examined, and the authors were contacted to obtain the necessary data.

2.2. Study Inclusion Criteria. The following stringent inclusion criteria were used: (1) all studies evaluated the relationship between warfarin maintenance dosage and NQO1 polymorphism and (2) information on the warfarin maintenance dosage (mean and standard deviation, SD) and sample size for each NQO1 genotype group was reported. It was not strictly required for patient characteristics (indication of warfarin, other interacting drugs, target International Normalized Ratio range, age, ethnicity, sex, etc.) to be reported in all studies.

2.3. Study Exclusion Criteria. Exclusion criteria included the following: (1) the publication types included review arti- cles, abstracts, conference proceedings, and case reports; (2) unavailable data on mean warfarin dosage and SD and sample size for each NQO1 genotype group; and (3) republished articles.

2.4. Data Extraction. Data were independently extracted, including clinical characteristics (such as name of the first author, publication year, sample size, target International Normalized Ratio range, indication of warfarin, predominant ethnicity, sex ratio, and mean age), sample size, allele frequencies, and warfarin maintenance dosage (mean and standard deviation, SD) for each NQO1 genotype by two reviewers (TLH and ZBR). For inconsistent information, the two reviewers retrieved and discussed the original data to reach a consensus.

2.5. Study Quality Assessment. The quality assessment of the included studies was performed by two reviewers (TLH and XPP) independently. The Newcastle-Ottawa Scale was applied to assess the quality of all studies [27]. Each study was assigned a score of $0-9$, and the studies were defined as high quality when the score was $\geq 7$.

2.6. Statistical Analysis. In the meta-analysis, NQO1 rs1800566 CT or TT carriers were defined as "rs1800566 T carriers," whereas NQO1 rs10517 AG or GG carriers were defined as "NQO1 rs10517 G carriers." Review Manager software version 5.4 was used to analyze the relationship between NQO1 gene polymorphisms and the warfarin maintenance dosage. We used the inverse variance method to weight each study, and the mean difference (MD) was used to determine the effect of each NQO1 genotype on the mean daily warfarin dosages (MDWD). To calculate the weighted mean difference (WMD), each MD was multiplied by the relative weight of each study. The sum of the WMD in each comparison was calculated to obtain the total WMD. In the meta-analysis, the $Z$ test was applied to examine the influence of NQO1 polymorphism on the warfarin maintenance dosage. The effect of the NQO1 genotype was considered as significant when the $P$ value was less than 0.05 . The means of the Cochran's $Q$ test (Mantel-Haenszel chi-squared test) was used to test heterogeneity in each genetic comparison group in the meta-analysis, and the results were expressed using the $P$ value and $I^{2}$ value. When the $P$ value $\geq 0.1$ or $I^{2}$ value $\leq$ $25 \%$, homogeneity was considered. A fixed effect model was also used to calculate the total WMD; otherwise, a randomeffect model was selected.

To eliminate the source of heterogeneity, sensitivity analyses were performed by deselecting studies individually in a certain order. Moreover, Begg's test [28] and Egger's test [29] were applied to assess publication bias in each metaanalysis.

\section{Results}

3.1. Study Identification and Characteristics. A schematic of the literature screening process is shown in Figure 1. A total of 124 articles were screened, and four articles describing 757 patients were included in the meta-analysis $[9,25,30,31]$. All included studies were published in English between 2007 


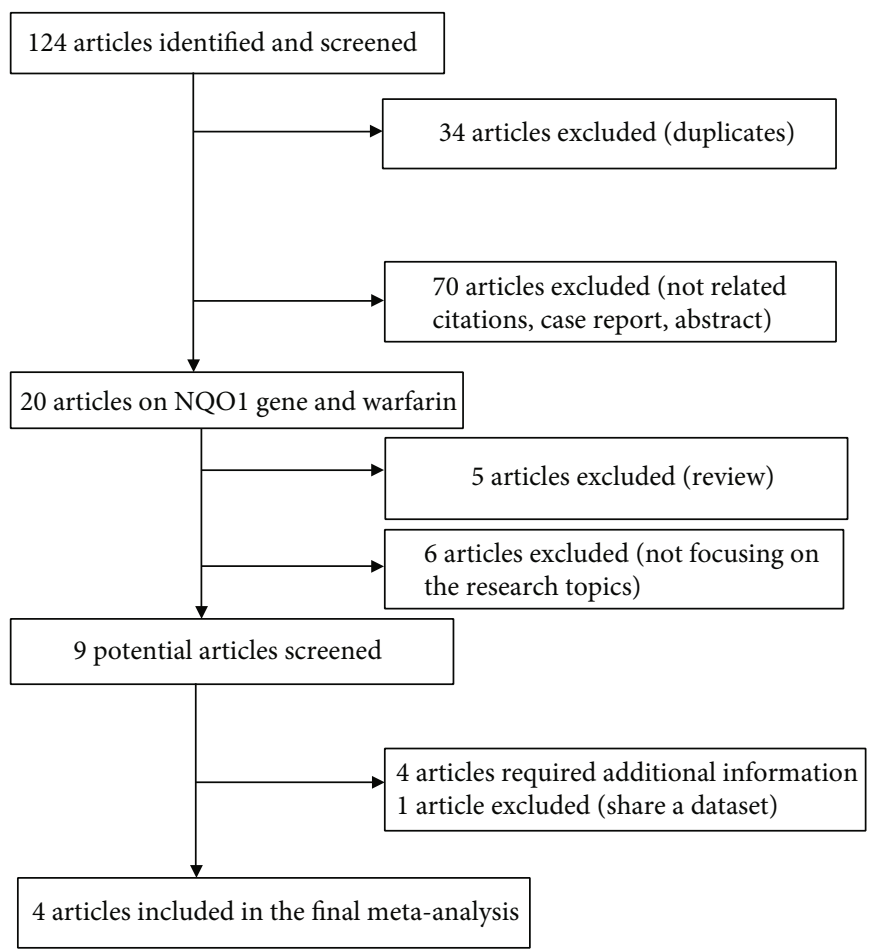

FIGURE 1: Flow diagram of the literature screening process used for the meta-analysis of NQO1 polymorphisms and warfarin maintenance dosage.

and 2020. Only one article referred to African-Americans [25]. The other included studies referred to Asian populations [9, 30, 31]. Moreover, only two studies focused on the relationship between NQO1 rs10517 genotypes and MDWDs $[9,30]$. According to the Newcastle-Ottawa Scale checklist, all studies with scores of 7 stars were considered as high quality. The characteristics of the four studies used in the metaanalysis are shown in Table 1.

\subsection{Meta-Analysis}

3.2.1. Relationship between NQO1 rs1800566 and Warfarin Maintenance Dosage. There were four articles [9, 25, 30, 31] included in this meta-analysis, and the results are shown in Figure 2. Compared with the NQO1 rs1800566 homozygous genotype (CC carriers), the warfarin dose in patients with the CT genotype was $13 \%$ higher $(P$ value $=0.01$, 95\% confidence interval (CI) $0.03-0.22$ ). Analysis using a fixed-effect model revealed homogeneity. No significant difference was found in the other comparisons (TT versus CC: $P=0.89$; T carriers versus CC: $P=0.39$; CT versus TT: $P=0.88$ ), and a random-effect model was applied in the other comparison of NQO1 rs1800566 genotype groups as significant heterogeneity was detected (all $P$ values $<0.1, I^{2}$ value $>50 \%$ ).

\subsubsection{Relationship between NQO1 rs10517 and Warfarin} Maintenance Dosage. Only two studies $[9,30]$ were included in this meta-analysis, which included 420 patients. Because of the limited number of patients with each genotype, we only conducted comparisons between NQO1 rs10517 G carriers and AA carriers. The results are shown in Figure 3. Com- pared with the AA carriers, $G$ carriers required significantly higher MDWD values $(P=0.001)$. Homogeneity was observed in the meta-analysis $\left(P=0.46, I^{2}=0 \%\right)$, and thus, a fixed-effect model was used.

3.3. Sensitivity Analysis. Sensitivity analyses were performed by individually deselecting studies. No significant change was found in the comparison between NQO1 rs10517 G carriers and AA carriers, whereas a significant change was found in comparisons of NQO1 rs1800566 (CT versus CC; TT versus CC; T carriers versus $\mathrm{CC}$ ). Researchers have suggested that NQO1 significantly influences the warfarin maintenance dosage in some Asian populations [15]; therefore, we removed one study that was not conducted in Asian populations [25] and performed a meta-analysis on NQO1 rs1800566 and warfarin maintenance dosage in Asian populations. There was no difference between Asian and all populations (data shown in Table 2).

3.4. Publication bias. Begg's and Egger's tests were applied in each meta-analysis. No publication bias was found in the association between NQO1 polymorphisms and warfarin dosage (data shown in Table 3).

\section{Discussion}

NQO1 is thought to be one of the gene polymorphisms influencing warfarin maintenance dosage [14-16]. In the vitamin $\mathrm{K}$ cycle, vitamin $\mathrm{K}$ is reduced to vitamin $\mathrm{K}$ hydroquinone by $\mathrm{NAD}(\mathrm{P}) \mathrm{H}$ :quinone oxidoreductase 1 (NQO1). The ability of NQO1enzyme activities was affected by the NQO1 


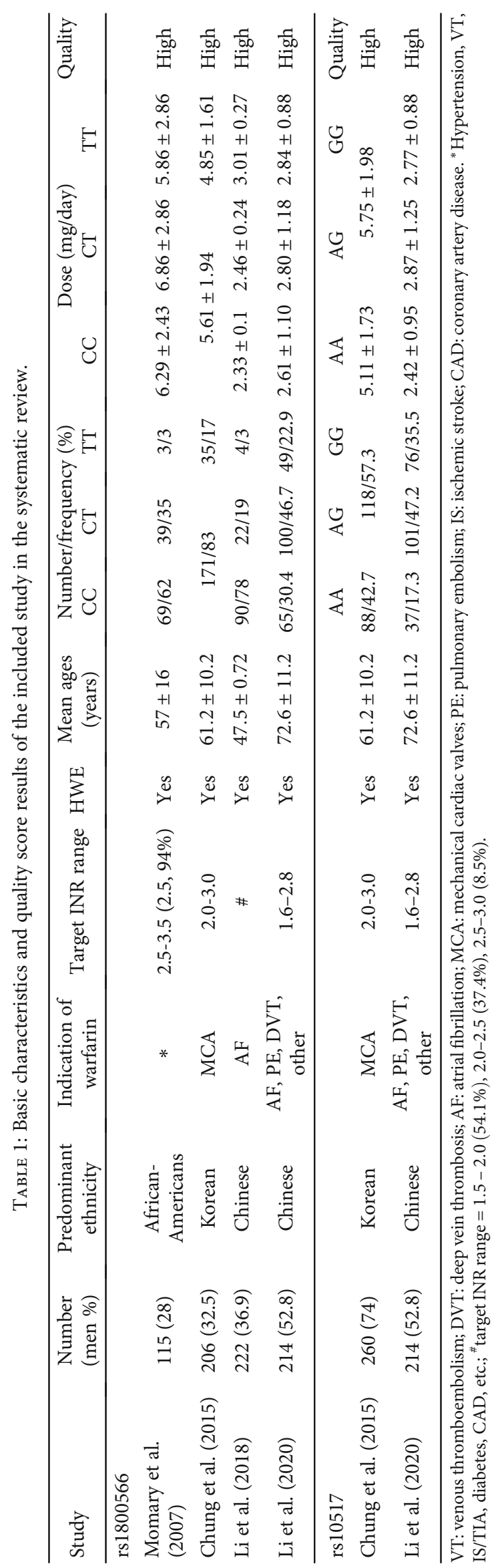




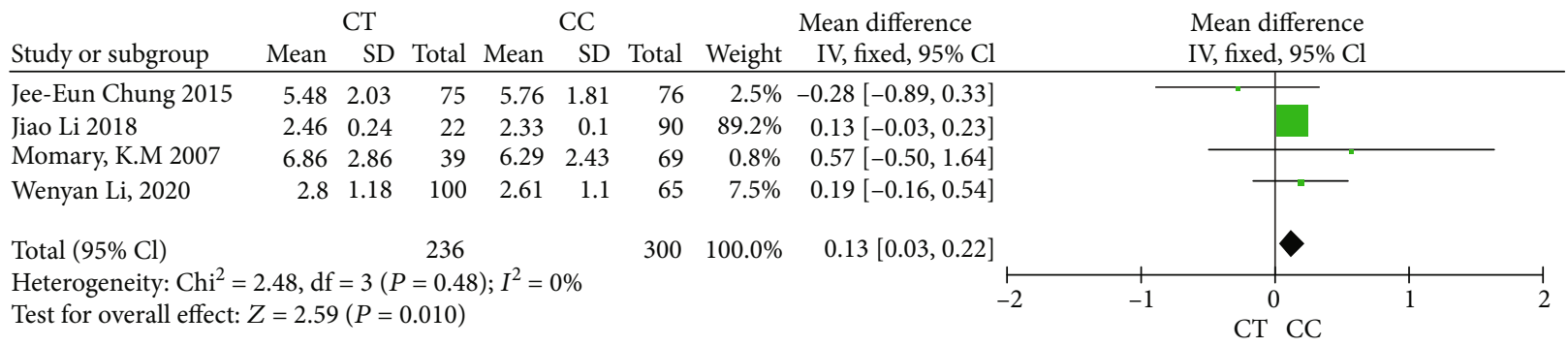

(a)

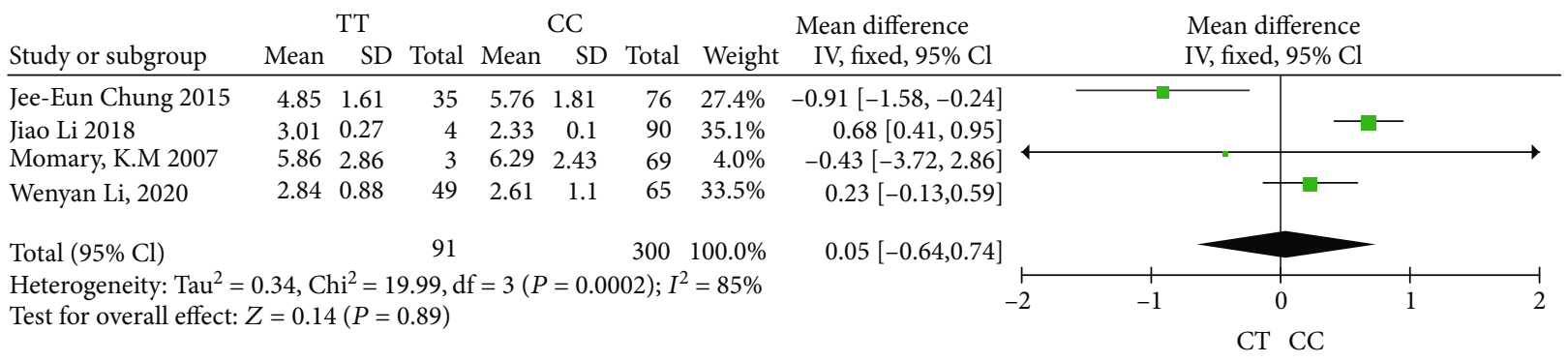

(b)

\begin{tabular}{|c|c|c|c|c|c|c|c|c|c|c|c|}
\hline \multirow[b]{2}{*}{ Study or subgroup } & \multicolumn{3}{|c|}{$\mathrm{CT}+\mathrm{TT}$} & \multicolumn{3}{|c|}{ CC } & \multicolumn{2}{|r|}{ Mean difference } & \multicolumn{2}{|c|}{ Mean difference } & \\
\hline & Mean & SD & Total & Mean & SD & Total & Weight & IV, fixed, $95 \% \mathrm{Cl}$ & & $95 \% \mathrm{Cl}$ & \\
\hline Jee-Eun Chung 2015 & 5.31 & 1.94 & 130 & 5.76 & 1.81 & 76 & $16.7 \%$ & $-0.45[-0.98,0.08]$ & & & \\
\hline Jiao Li 2018 & 2.54 & 0.31 & 26 & 2.33 & 0.1 & 90 & $48.0 \%$ & $0.21[0.09,0.33]$ & & -1 & \\
\hline Momary, K.M 2007 & 6.79 & 2.84 & 42 & 6.29 & 2.43 & 69 & $5.7 \%$ & $0.50[-0.53,1.53]$ & & & \\
\hline Wenyan Li, 2020 & 2.81 & 1.09 & 149 & 2.61 & 1.1 & 65 & $29.6 \%$ & $0.20[-0.12,0.52]$ & & & \\
\hline Total $(95 \% \mathrm{Cl})$ & & & 347 & & & 300 & $100.0 \%$ & $0.11[-0.15,0.37]$ & & & \\
\hline $\begin{array}{l}\text { Heterogeneity: } \mathrm{Tau}^{2}= \\
\text { Test for overall effect: }\end{array}$ & $\begin{array}{l}0.03, \mathrm{Cl} \\
\mathrm{Z}=0.85\end{array}$ & $\begin{array}{l}\mathrm{ai}^{2}=6 \\
(P=\end{array}$ & $\begin{array}{l}.12, \mathrm{df} \\
0.39)\end{array}$ & $=3(P$ & .1 & $; I^{2}=5$ & & $\begin{array}{r}- \\
-2\end{array}$ & -1 & 0 & 1 \\
\hline
\end{tabular}

(c)

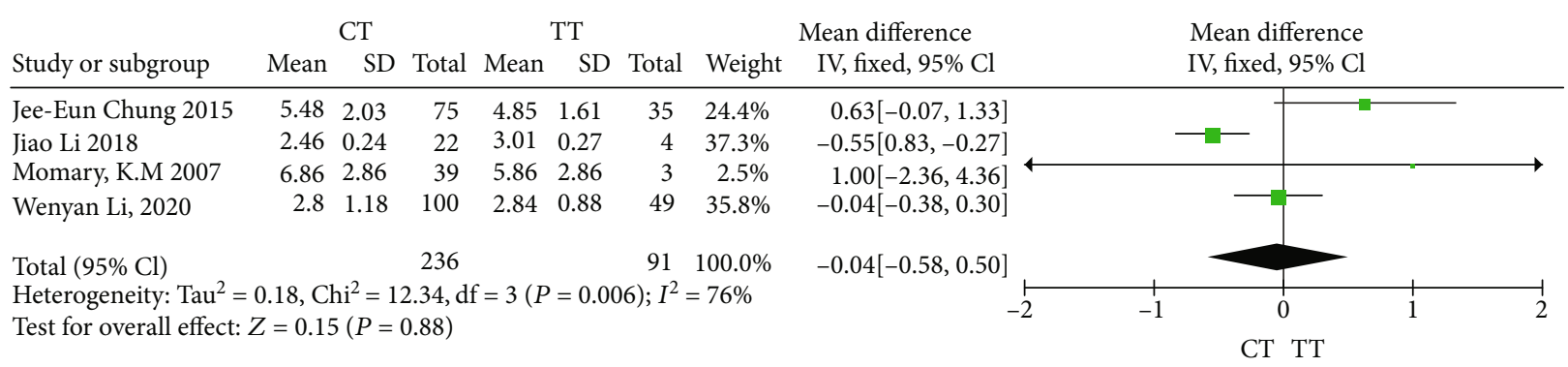

(d)

FIGURE 2: Forest plots illustrating the influence of NQO1 rs1800566 polymorphisms on warfarin dose requirement. (a) Relative warfarin dosage requirement of rs1800566 CT carriers compared to CC carriers (wild-type). (b) Relative warfarin dosage requirement of rs1800566 TT carriers versus CC carriers. (c) Relative warfarin dosage requirement of rs1800566 T carriers (CT or TT carriers) versus CC carriers. (d) Relative warfarin dosage requirement of rs1800566 CT carriers versus TT carriers. Mean (SD): mean and standard deviation of normalized warfarin doses associated with each genotype. IV: inverse variance; CI: confidence interval. Values in the square brackets denote $95 \%$ confidence intervals.

genotype, especially NQO1 rs1800566 [32]. When a C was replaced by $\mathrm{T}$ at position 609 of the cDNA, the proline was replaced by the serine at amino acid 187 of the functional protein, leading to a possible loss of external interactions with other moieties necessary for its proper functioning [32]. It was proven that individuals with the NQO1 rs1800566 variant homozygote have no NQO1 activity, and individuals who are NQO1 rs180056 heterozygous have low-to-intermediate NQO1 activity compared to wild-type ones [18, 33]. Warfarin exerts its action by interfering with the conversion of vitamin $\mathrm{K}$ epoxide to vitamin $\mathrm{K}$ in the vitamin $\mathrm{K}$ cycle, leading to reduced activation of vitamin $\mathrm{K}$ dependent clotting factors [19]. Therefore, NQO1 probably affects warfarin maintain dosage.

Previous meta-analyses on the role of CYP2C9, VKORC1, and CYP4F2 polymorphisms in the warfarin maintenance 


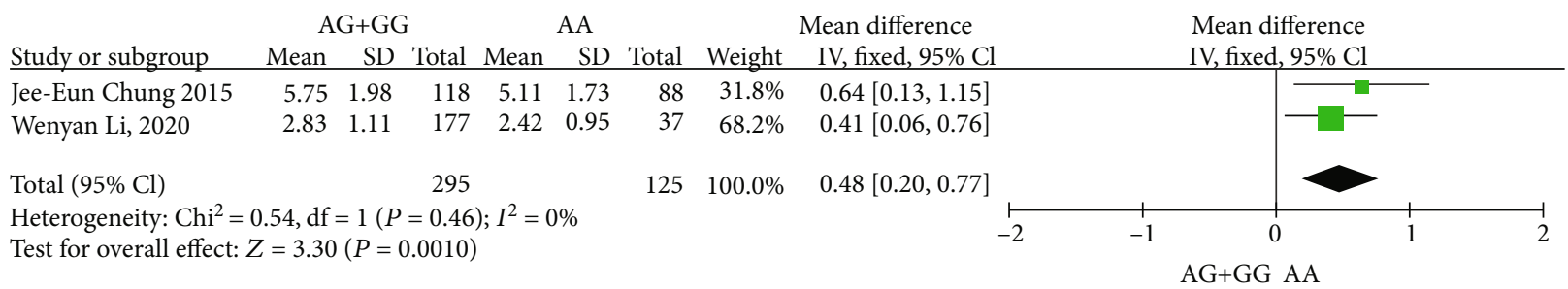

FIGURE 3: Forest plots showing the influence of NQO1 rs10517 on warfarin maintenance dosage. Relative warfarin dosage requirement of NQO1 rs10517 G carriers (GA or GG carriers) compared to AA carriers (wild-type). Mean (SD): mean and standard deviation of normalized warfarin doses associated with each genotype. WMD: weighted mean differences; CI: confidence interval.

TABLE 2: The mean values of warfarin dose for each genotype and comparison of the effect of variant NQO1 rs1800566 genotypes on warfarin dosage requirement in Asian and all populations.

\begin{tabular}{|c|c|c|c|c|c|c|}
\hline \multirow{2}{*}{ Groups } & \multicolumn{2}{|c|}{ All population } & \multirow{2}{*}{$P$ value } & \multicolumn{2}{|c|}{ Asian population } & \multirow{2}{*}{$P$ value } \\
\hline & MDWD & WMD (95\% CI) & & MDWD & WMD & \\
\hline CC & $4.17 \pm 2.38$ & - & - & $3.54 \pm 1.96$ & - & - \\
\hline CT & $4.29 \pm 2.53$ & - & - & $3.78 \pm 2.12$ & - & - \\
\hline $\mathrm{TT}$ & $3.72 \pm 1.63$ & - & - & $3.65 \pm 1.55$ & - & - \\
\hline CT vs. CC & - & $13 \%(3,22 \%)$ & 0.01 & - & $12 \%(3,22 \%)$ & 0.01 \\
\hline TT vs. CC & - & $5 \%(-64 \%, 74 \%)$ & 0.89 & - & $7 \%(-66 \%, 79 \%)$ & 0.86 \\
\hline CT vs. TT & - & $-4 \%(-58 \%, 50 \%)$ & 0.88 & - & $-6 \%(-63 \%, 50 \%)$ & 0.82 \\
\hline T carriers vs. CC & - & $11 \%(-15 \%, 37 \%)$ & 0.39 & - & $8 \%(-21 \%, 37 \%)$ & 0.60 \\
\hline
\end{tabular}

MDWD: mean daily warfarin dosages; WMD: weighted mean differences; CI: confidence interval; the inverse variance method was used in all comparisons of weight in each study.

TABLE 3: Begg's test for each meta-analysis.

\begin{tabular}{lcc}
\hline SNP & Begg's test \\
\hline rs10517 & G carriers versus AA & $\operatorname{Pr}>|z|=1.000$ \\
\hline CT versus CC & $\operatorname{Pr}>|z|=0.734$ \\
rs1800566 & TT versus CC & $\operatorname{Pr}>|z|=0.734$ \\
& CT versus TT & $\operatorname{Pr}>|z|=0.734$ \\
& T carriers versus CC & $\operatorname{Pr}>|z|=0.734$ \\
\hline
\end{tabular}

dosage have been conducted $[4,6,34,35]$. However, these studies did not completely explain the variation in therapeutic warfarin dosages $[14,16]$. NQO1 genotypes could be one factor which could affect warfarin dosage [21, 23], whereas the relationship between NQO1 genotypes and warfarin maintenance dosage was controversial. In 2012, Bress et al. confirmed that NQO1 rs1800566 genotypes significantly affected the warfarin-required dosage and predicted that patients with variant genotypes required higher warfarin dosages [14]. Chung et al. showed that patients with NQO1 rs1800566 variant genotypes required lower warfarin maintenance dosages; patients with NQO1 rs10517 variant genotypes required higher warfarin maintenance dose compared with those who were wild-type homozygotes [9]. However, some studies found no significant association between NQO1 polymorphism and the warfarin maintenance dosage requirements $[30,31]$. Although Asiimwe et al. verified that
NQO1 rs1800566 has no significant influence on warfarin dosage in Black-African patients by meta-analysis, the role of NQO1 genotypes in warfarin maintenance in other ethnic groups was not investigated, the relationship between other NQO1 genotypes and warfarin dosages was not researched, and the study sample size was small [36]. Until now, the relationship between NQO1 genotypes and warfarin dosage remains unclear, especially in non-Black-African patients. Therefore, we conducted a meta-analysis to analyze the relationship between NQO1polymorphisms (rs1800566 and rs10517) and the warfarin maintenance dosage.

We found that NQO1 rs10517 G carriers required $48 \%$ higher MDWD than AA carriers. In patients with NQO1 rs 1800566, those with the CT genotype required 13\% higher MDWD than those with the CC genotype, which was significant, whereas no significant difference was found in the other comparisons (TT versus CC; T carriers versus CC; CT versus TT).

In our meta-analysis, we selected two single nucleotide polymorphisms (SNPs) of the NQO1 gene, rs1800566 and rs10517, which have proven to be significantly associated with warfarin maintenance doses. However, our results showed that the effects of two SNPs on warfarin maintenance dosages were inconsistent. This may be due to the differing mechanism of the two SNPs on warfarin. Rs10517 in the $3^{\prime}$ UTR region of the NQO1 gene might alter the function of the protein by affecting the secondary structure of mRNA and upregulating the coagulation factor activity [37], whereas 
NQO1 rs1800566 affects vitamin K metabolism and alters warfarin response [9].

Sensitivity analyses performed by deselecting studies individually in a certain order revealed no significant change in the comparison between NQO1 rs10517 G carriers and AA carriers, suggesting that our results were stable and reliable. Significant changes were found in the comparisons of the NQO1 rs1800566 genotype (CT versus CC; TT versus CC; T carriers versus CC). Therefore, the results of the NQO1 rs1800566 genotype comparisons must be considered with caution.

It is known that the frequency of VKORC1 and CYP2C9 significantly differs in different ethnic groups, and the contributions of the VKORC1 and CYP2C9 genotypes to warfarin dose requirements are inconsistent in different ethnic populations [15]. Additionally, it is thought that NQO1 influences the warfarin maintenance dosage significantly in some Asian populations [15]; therefore, we removed the study in African-Americans [25] and conducted a metaanalysis of NQO1 rs1800566 and the warfarin maintenance dosage in Asian populations. However, compared with all populations, the results showed no significant difference, possibly because of the limited number of articles and sample size of NQO1 rs1800566. No Caucasian population was included in the meta-analysis. Thus, the influence of NQO1 genotypes on the warfarin dosage in Caucasians is unclear in our meta-analysis.

Our meta-analysis showed that patients with the NQO1 rs1800566 CT genotype required a higher warfarin dosage than those with the CC genotype (wild-type genotype), and the level of evidence of NQO1 rs1800566 for warfarin dosage is grade 3(available at http://www.pharmgkb.org/). However, the result was in contrast to the findings of previous studies $[17,24]$. This may be because the NQO1 variant allele was likely in linkage disequilibrium with the functional singlenucleotide polymorphism in some populations [15]. Therefore, studies are required to focus on the underlying reason for the NQO1 association. In addition, no significant difference was found in the other comparisons of NQO1 rs1800566 (TT versus CC; T carriers versus CC; CT versus TT), possibly because the number of included studies and sample size of patients with the NQO1 rs1800566 genotype was small. Although patients with the NQO1 rs10517 variant genotypes (AG carriers and GG carriers) required higher warfarin maintenance doses than those with the wild-type homozygote (AA carriers) genotype in our meta-analysis, the effect of the NQO1 rs10517 genotype on warfarin should be considered with caution because the number of included studies was limited. Further analysis of the relationship between NQO1 genotypes (rs1800566 and rs10517) and the warfarin maintenance dosage is required to confirm our results.

Our study had some limitations. First, the number of articles used was small, including only four studies on NQO1 rs1800566 and two studies on NQO1 rs10517. Second, the studies only included patients of African-American and Asian descent. Third, some studies showed a significant effect between NQO1genotypes, and the warfarin dosages were not included because of lack of data. Further studies are needed to support our findings.

\section{Conclusions}

This meta-analysis focused on the relationship between the warfarin maintenance dosage and NQO1 polymorphism. We found that NQO1 rs1800566 CT carriers required a $13 \%$ higher warfarin dose compared to CC carriers, and no associations were observed in the other comparisons of NQO1 rs1800566 genotypes. NQO1 rs10517 G carriers required a $48 \%$ higher warfarin dose than AA carriers. NQO1 rs1800566 polymorphisms and NQO1 rs10517 polymorphisms may significantly affect individual warfarin dosage requirements. Additional studies of larger sample sizes in multiethnic populations are required to avoid selection bias and confirm our findings.

\section{Conflicts of Interest}

The authors declared no conflict of interest.

\section{Authors' Contributions}

Lihong Tian is the guarantor of the entire study. Lihong Tian handled the study concepts. Pingping Xiao handled the study design. Lihong Tian, Bingrong Zhou, Min Song, and Jianfeng Lin were in charge of literature research. Lihong Tian and Bingrong Zhou were in charge of the data acquisition. Lihong Tian, Yishan Chen, Qingqing Wang, and Lijuan Kang handled the data analysis/interpretation. Pingping Xiao and Qingxiu Wu were in charge of the statistical analysis. Lihong Tian, Min Song, and Jianfeng Lin were in charge of the manuscript preparation. Lihong Tian handled the manuscript definition of intellectual content. Lihong Tian handled the manuscript editing. Lihong Tian and Pingping Xiao were in charge of manuscript revision/review. Lihong Tian was in charge of the manuscript final version approval.

\section{Acknowledgments}

We would like to thank Editage (http://www.editage.cn/) for English language editing. This work was supported by the 2020 Young \& Middle-Aged Backbone Health Training Program of Fujian Province (No. 2020 GGB068) and Xiamen Medical and Health Guidance Project (No. 3502Z20199137).

\section{References}

[1] N. Oake, A. Jennings, A. J. Forster, D. Fergusson, S. Doucette, and C. van Walraven, "Anticoagulation intensity and outcomes among patients prescribed oral anticoagulant therapy: a systematic review and meta-analysis," CMAJ, vol. 179, no. 3, pp. 235-244, 2008.

[2] A. L. Jorgensen, R. J. FitzGerald, J. Oyee, M. Pirmohamed, and P. R. Williamson, "Influence of CYP2C9 and VKORC1 on patient response to warfarin: a systematic review and metaanalysis," PLoS One, vol. 7, no. 8, p. e44064, 2012.

[3] R. Liang, C. Wang, H. Zhao, J. Huang, D. Hu, and Y. Sun, "Influence of CYP4F2 genotype on warfarin dose requirement-a systematic review and meta-analysis," Thrombosis Research., vol. 130, no. 1, pp. 38-44, 2012. 
[4] M. Takeuchi, T. Kobayashi, T. Biss et al., "_CYP2C9_, _VKORC1_, and _CYP4F2_ polymorphisms and pediatric warfarin maintenance dose: a systematic review and metaanalysis," The Pharmacogenomics Journal, vol. 20, no. 2, pp. 306-319, 2020.

[5] Y. Sun, Z. Wu, S. Li et al., "Impact of gamma-glutamyl carboxylase gene polymorphisms on warfarin dose requirement: a systematic review and meta-analysis," Thrombosis Research, vol. 135, no. 4, pp. 739-747, 2015.

[6] L. Tian, J. Zhang, S. Xiao, J. Huang, Y. Zhang, and J. Shen, "Impact of polymorphisms of the GGCX gene on maintenance warfarin dose in Chinese populations: systematic review and meta-analysis," Meta gene., vol. 5, pp. 43-54, 2015.

[7] J. Zhang, L. Tian, Y. Zhang, and J. Shen, "The influence of _VKORC1_ gene polymorphism on warfarin maintenance dosage in pediatric patients: A systematic review and metaanalysis," Thrombosis Research, vol. 136, no. 5, pp. 955-961, 2015.

[8] A.-K. Hamberg and M. Wadelius, "Pharmacogenetics-based warfarin dosing in children," Pharmacogenomics, vol. 15, no. 3, pp. 361-374, 2014.

[9] J.-E. Chung, B. C. Chang, K. E. Lee, J. H. Kim, and H. S. Gwak, "Effects of $\mathrm{NAD}(\mathrm{P}) \mathrm{H}$ quinone oxidoreductase 1 polymorphisms on stable warfarin doses in Korean patients with mechanical cardiac valves," European Journal of Clinical Pharmacology, vol. 71, no. 10, pp. 1229-1236, 2015.

[10] T. T. Biss, P. J. Avery, L. R. Brandão et al., "VKORC1 and CYP2C9 genotype and patient characteristics explain a large proportion of the variability in warfarin dose requirement among children," Blood, vol. 119, no. 3, pp. 868-873, 2012.

[11] L. K. Teh, I. M. Langmia, M. H. Fazleen Haslinda et al., "Clinical relevance of VKORC1 (G-1639A and C1173T) and CYP2C9*3 among patients on warfarin," Journal of Clinical Pharmacy and Therapeutics, vol. 37, no. 2, pp. 232-236, 2012.

[12] J. A. Johnson, L. Gong, M. Whirl-Carrillo et al., "Clinical pharmacogenetics implementation consortium guidelines for CYP2C9 and VKORC1 genotypes and warfarin dosing," Clinical Pharmacology \& Therapeutics, vol. 90, no. 4, pp. 625-629, 2011.

[13] G. M. Cooper, J. A. Johnson, T. Y. Langaee et al., “A genomewide scan for common genetic variants with a large influence on warfarin maintenance dose," Blood, vol. 112, no. 4, pp. 1022-1027, 2008.

[14] A. Bress, S. R. Patel, M. A. Perera, R. T. Campbell, R. A. Kittles, and L. H. Cavallari, "Effect of NQO1 and CYP4F2 genotypes on warfarin dose requirements in Hispanic-Americans and African-Americans," Pharmacogenomics, vol. 13, no. 16, pp. 1925-1935, 2012.

[15] N. el Rouby, L. Rodrigues Marcatto, K. Claudio et al., "Multisite investigation of genetic determinants of warfarin dose variability in Latinos," Clinical and Translational Science, vol. 14, no. 1, pp. 268-276, 2021.

[16] Z. Luo, X. Li, M. Zhu et al., "Identification of novel variants associated with warfarin stable dosage by use of a two-stage extreme phenotype strategy," Journal of Thrombosis and Haemostasis, vol. 15, no. 1, pp. 28-37, 2017.

[17] D. Ross and D. Siegel, "NAD $(\mathrm{P}) \mathrm{H}$ :quinone oxidoreductase 1 (NQO1, DT-diaphorase), functions and pharmacogenetics," Methods in Enzymology, vol. 382, pp. 115-144, 2004.

[18] D. Siegel, S. M. McGuinness, S. L. Winski, and D. Ross, “Genotype-phenotype relationships in studies of a polymorphism in
NAD(P)H:quinone oxidoreductase 1," Pharmacogenetics, vol. 9, no. 1, pp. 113-122, 1999.

[19] M. Wadelius and M. Pirmohamed, "Pharmacogenetics of warfarin: current status and future challenges," The Pharmacogenomics Journal, vol. 7, no. 2, pp. 99-111, 2007.

[20] L. H. J. de Haan, A. M. J. F. Boerboom, I. M. C. M. Rietjens et al., "A physiological threshold for protection against menadione toxicity by human $\mathrm{NAD}(\mathrm{P}) \mathrm{H}$ :quinone oxidoreductase (NQO1) in Chinese hamster ovary (CHO) cells," Biochemical Pharmacology, vol. 64, no. 11, pp. 1597-1603, 2002.

[21] R. Wallin, "Vitamin K-dependent carboxylation in the developing rat: evidence for a similar mechanism of action of warfarin in fetal and adult livers," Pediatric Research, vol. 26, no. 4, pp. 370-376, 1989.

[22] R. Wallin and L. F. Martin, "Vitamin K-dependent carboxylation and vitamin K metabolism in liver. Effects of warfarin," Journal of Clinical Investigation, vol. 76, no. 5, pp. 1879$1884,1985$.

[23] R. M. L. Wallin and L. F. Martin, "Warfarin poisoning and vitamin $\mathrm{K}$ antagonism in rat and human liver. Design of a system in vitro that mimics the situation in vivo," The Biochemical Journal, vol. 241, no. 2, pp. 389-396, 1987.

[24] H.-Y. Shyu, C. S. Fong, Y. P. Fu et al., "Genotype polymorphisms of_GGCX_,_NQO1_, and_VKORC1_genes associated with risk susceptibility in patients with large-artery atherosclerotic stroke," Clinica Chimica Acta, vol. 411, no. 11-12, pp. 840-845, 2010.

[25] K. M. Momary, N. L. Shapiro, M. A. G. Viana, E. A. Nutescu, C. M. Helgason, and L. H. Cavallari, "Factors influencing warfarin dose requirements in African-Americans," Pharmacogenomics, vol. 8, no. 11, pp. 1535-1544, 2007.

[26] M. Wadelius, L. Y. Chen, N. Eriksson et al., "Association of warfarin dose with genes involved in its action and metabolism," Human Genetics, vol. 121, no. 1, pp. 23-34, 2007.

[27] G. A. Wells, B. Shea, D. O'Connell et al., "The NewcastleOttawa Scale (NOS) for assessing the quality of nonrandomized studies in meta-analysis," Ottawa Hospital Research Institute Web site, vol. 7, 2014.

[28] C. B. Begg and M. Mazumdar, "Operating characteristics of a rank correlation test for publication bias," Biometrics, vol. 50, no. 4, pp. 1088-1101, 1994.

[29] M. Egger, G. D. Smith, M. Schneider, and C. Minder, "Bias in meta-analysis detected by a simple, graphical test," $B M J$, vol. 315, no. 7109, pp. 629-634, 1997.

[30] W. Li, P. Zhao, L. Chen et al., "Impact ofCYP2C9,VKORC1,ApoEandABCB1polymorphisms on stable warfarin dose requirements in elderly Chinese patients," Pharmacogenomics, vol. 21, no. 2, pp. 101-110, 2020.

[31] J. Li, W. Yang, Z. Xie, K. Yu, Y. Chen, and K. Cui, "Impact of VKORC1, CYP4F2 and NQO1 gene variants on warfarin dose requirement in Han Chinese patients with catheter ablation for atrial fibrillation," BMC CardiovascDisord., vol. 18, no. 1, p. 96, 2018.

[32] Z. Basharat, A. Messaoudi, S. Ruba, and A. Yasmin, "NQO1 rs1800566 polymorph is more prone to NOx induced lung injury: endorsing deleterious functionality through informatics approach," Gene, vol. 591, no. 1, pp. 14-20, 2016.

[33] X. Gong, R. Gutala, and A. K. Jaiswal, "Quinone oxidoreductases and vitamin K metabolism," in Vitamins \& Hormones, pp. 85-101, Elsevier, 2008. 
[34] J. D. Lindh, L. Holm, M. L. Andersson, and A. Rane, "Influence of CYP2C9 genotype on warfarin dose requirements-a systematic review and meta-analysis," European Journal of Clinical Pharmacology, vol. 65, no. 4, pp. 365-375, 2009.

[35] L. Yang, W. Ge, F. Yu, and H. Zhu, "Impact of _VKORC1_ gene polymorphism on interindividual and interethnic warfarin dosage requirement - A systematic review and meta analysis," Thrombosis Research, vol. 125, no. 4, pp. e159-e166, 2010.

[36] I. G. Asiimwe, E. J. Zhang, R. Osanlou et al., "Genetic factors influencing warfarin dose in black-African patients: a systematic review and meta-analysis," Clinical Pharmacology \& Therapeutics, vol. 107, no. 6, pp. 1420-1433, 2020.

[37] A. Valinezhad Orang, R. Safaralizadeh, and M. KazemzadehBavili, "Mechanisms of miRNA-mediated gene regulation from common downregulation to mRNA-specific upregulation," International journal of genomics., vol. 2014, article 970607, pp. 1-15, 2014. 